\title{
Localized Multi-Photon Photoelectron Emission From Indium Tin Oxide in Proximity to Gold in Nanostructured Gold-ITO-Glass Films
}

\author{
R. C. Word,* T. Dornan, * and R. Könenkamp* \\ *Department of Physics, Portland State University, PO Box 751, Portland, Oregon, 97207
}

In typically understood photoemission the absorption of a single photon of high enough energy $h v>$ W permits an electron to escape the surface of a material. However, under certain conditions the photoemission may occur via the absorption of more than one photon of energy $h v<\mathrm{W}$. The efficiency of multi-photon photoemission is poor, but can be enhanced by several orders of magnitude in the presence of the strong fields of localized surface plasmons (LSP) or "hotspots." Surface plasmons (SP) are collective electron oscillations at the metal/dielectric interfaces that are coupled to optical fields of the same frequency. The most efficient method of generating SPs is by attenuated total internal reflection in the Otto or Kretschamann configurations. However, scattering from sufficiently random geometries of isolated metal structures can lead to LSPs [1]. These, for example, can be visualized via photoemission microscopy (PEEM) as bright spots on nano-scale metals [2]. For some geometries strong fields can exist outside the metal [3]. In this paper, we report 3 -photon photoelectron emission from ITO in the vicinity of gold in nanostructured gold-ITO films.

The nanostructured gold films were milled from a $\sim 100-\mathrm{nm}$ film of gold thermally evaporated onto a $0.2-\mathrm{mm}$ thick glass cover slip pre-coated with $\sim 60-\mathrm{nm}$ layer of ITO (SPI Supplies). The gold was milled with an array of $10 \times 10-\mu \mathrm{m}$ squares using the 100-pA gallium beam of a dual-beam FIB. The Ga exposure time of each square differed as shown in Fig. 1a. The specimen is backed by the polished stainless steel of the specimen holder, which reflects light transmitted through the gold-ITO back towards the glass side of the ITO-gold film.

Our PEEM is equipped with a 100-W Hg arc lamp, that has a strong UV emission line at $254 \mathrm{~nm}$, a 244-nm 100-mW CW Ar laser, and a frequency doubled Ti-sapphire laser with 100-fs pulse duration and $80 \mathrm{MHz}$ pulse frequency. The $\mathrm{CW}$ output power of the fs laser is 300 to $950 \mathrm{~mW}$ and is wavelength-tunable from 780 to $920 \mathrm{~nm}$. The fs laser is focused to a spot about $100-\mu \mathrm{m}$ in diameter. Each light source is incident on the specimen at $60^{\circ}$ to the normal. The configuration of light sources allows simultaneous use of the $\mathrm{Hg}$ arc lamp and the fs laser. This grants the ability to precisely determine the location of the hotspots.

With exposure to $780-880 \mathrm{~nm}$ fs-laser light the gold-ITO film generates a wealth of hotspots. Photoemission was not strongly wavelength dependent, but was most efficient at $790 \mathrm{~nm}$ where we found $\sim 100$ hotspots in one particular $10 \times 10-\mu \mathrm{m}$ milled square. The overwhelming majority hotspots were in regions of heavy Ga milling (Fig. 2b and 2c). Here, irregular structures of gold in the order of $100-\mathrm{nm}$ wide and 0.1 to $1 \mu \mathrm{m}$ long are separated by glass depressions. As observed from above, the gold is further separated from the glass by a thin $\sim 10-\mathrm{nm}$ wide zone of exposed ITO. Comparison of high resolution PEEM and SEM images taken of the same areas, we find that the great majority of hotspots are on this zone of exposed ITO (Fig 2a). Furthermore, in some areas, ITO forms "bridges" between gold structures (Fig. 2b). On these bridges, we find some of the brightest hotspots. By integrating images of hotspots, we find that the photoelectron intensity varies with the third-power of incident laser light (Fig. 2c). This indicates a three-photon absorption process, as would be expected 
for 1.5-1.6-eV photon energies and ITO's work function of 4 to $5 \mathrm{eV}$. It is our conclusion that the photoemission hotspots are generated from ITO due to strong fields generated externally by LSPs in the neighboring gold nanostructures. [4]

\section{References}

[1] A.V. Zayats et al. J. Opt. A. 5 (2003) 816

[2] L. Douillard et al. Nano Lett. 8 (2008) 935

[3] L. Kuiru et al. Phys. Rev Lett. 91 (2003) 227402-1

[4] This research was partially supported by NSF under grant number DBI-0352224 and by DOE under DE-FG02-07ER46406

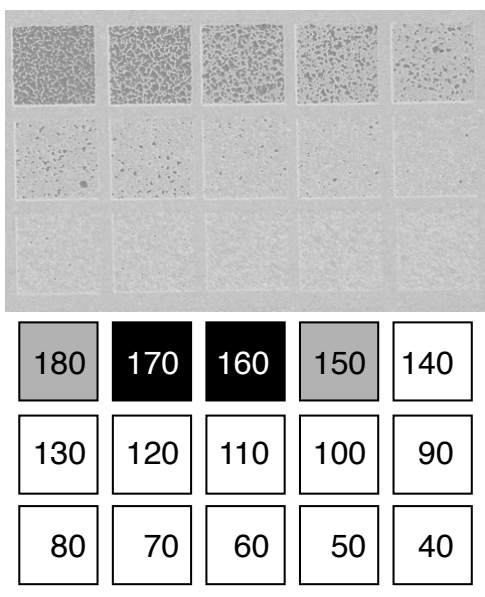

(a)

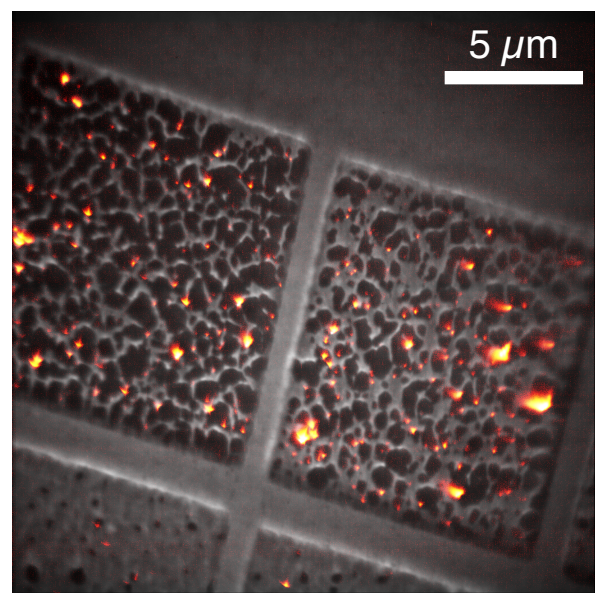

(b)

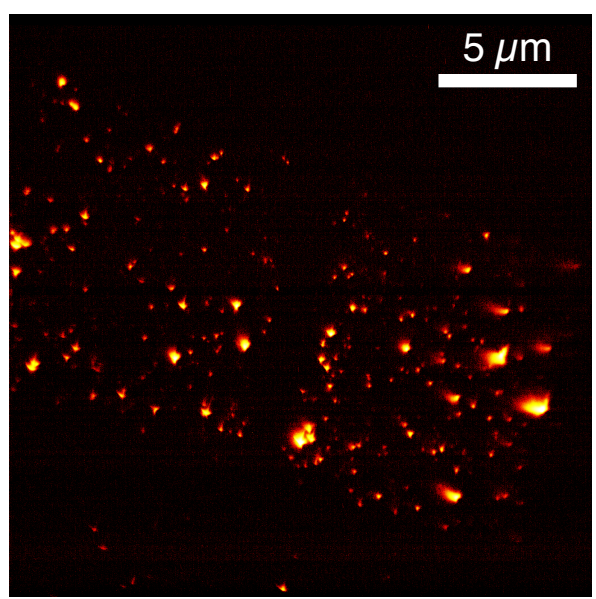

(c)

FIG. 1. (a) SEM micrograph of Ga-milled gold-ITO-glass film. Beam exposure time (in sec.) shown in squares below. Dark squares indicate areas with numerous hotspots. Black squares indicate areas shown in PEEM micrographs. (b) Composite PEEM micrograph of region illuminated by UV and 790-nm fs laser light. (c) Region illuminated 790-nm fs laser light showing numerous hotspots.

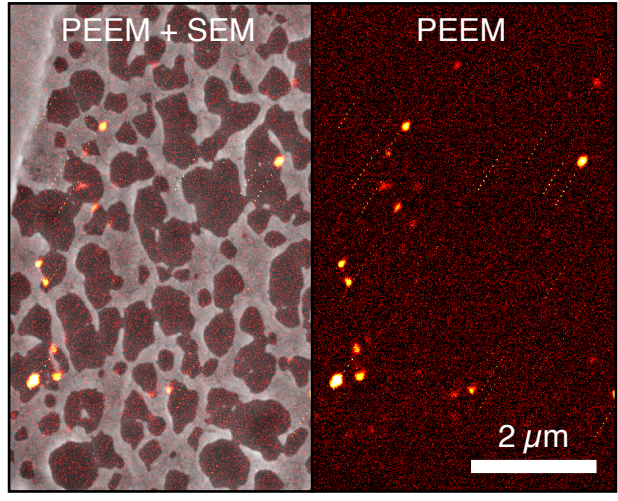

(a)

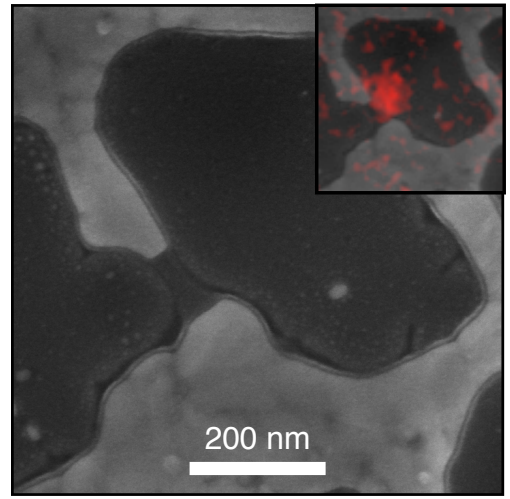

(b)

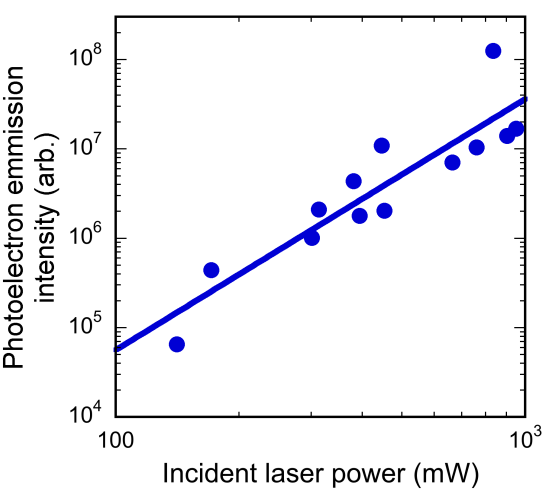

(c)

FIG. 2. (a) After alignment of PEEM and SEM images we find that hotspots originate from areas of exposed ITO. (b) SEM micrograph of milled gold-ITO-glass. ITO is visible as a $\sim 10$-nm wide dark band. (c) Intensity of hotspots is proportional to roughly the 3rd power of incident 790-nm fs laser power indicating a three-photon absorption process. 